Adriana Skamvetsakis ${ }^{\mathrm{a}}$

Rosemari Santia

Luiz Henrique Paim da Rocha ${ }^{\mathrm{a}}$

Fabiane Zardo Brettas ${ }^{\mathrm{a}}$

Patrícia de Souza Fagundes ${ }^{a}$

Maria Juliana Moura-Correa ${ }^{\mathrm{b}}$

a Centro Regional de Referência em Saúde do Trabalhador da Região dos Vales, Secretaria Municipal de Saúde, Prefeitura Municipal de Santa Cruz do Sul. Santa Cruz do Sul, RS, Brasil.

b Universidade Federal da Bahia, Instituto de Saúde Coletiva, Programa Integrado em Saúde Ambiental e Saúde do Trabalhador. Salvador, BA, Brasil.

Contato:

Adriana Skamvetsakis

E-mail:

adriana.cerest@santacruz.rs.gov.br

Este trabalho recebeu fomento do Chamamento Público nº 05/2014 Iniciativas educacionais aplicadas à Vigilância em Saúde da Secretaria de Vigilância em Saúde do Ministério da Saúde.

Os autores declaram que não há conflitos de interesses e que este estudo não teve como base tese ou dissertação.

Parte da experiência relatada neste trabalho foi oralmente apresentada no IV Encontro Estadual de Vigilância em Postos de Combustíveis Vulnerabilidades em postos de combustíveis - uma abordagem em defesa da vigilância da saúde dos trabalhadores, evento realizado pelo Centro de Vigilância Sanitária da Secretaria de Estado da Saúde de São Paulo, em 25 e 26 de novembro de 2014. Não houve publicação de anais.

Recebido: 21/12/2015

Revisado: $17 / 06 / 2016$

Aprovado: 22/06/2016

\section{Exposição ao benzeno em postos de combustíveis: estratégia de ações integradas de Vigilância em Saúde do Trabalhador na região dos Vales/RS}

\author{
Exposure to benzene in gas stations: strategy for integrated \\ actions in Occupational Health Surveillance in Região dos \\ Vales, Rio Grande do Sul, Brazil
}

\section{Resumo}

Introdução: O benzeno é uma importante fonte de contaminação ambiental e está presente na composição da gasolina, expondo trabalhadores de Postos de Revenda de Combustíveis (PRC) a intoxicações. Objetivo: relatar experiências de vigilância da exposição ao benzeno do Centro de Referência em Saúde do Trabalhador da região dos Vales/RS. Métodos: a vigilância no território incluiu o mapeamento regional dos PRC, ações intrasetoriais, intersetoriais e interinstitucionais, inspeção nos locais de trabalho, desenvolvimento de instrumento de intervenção específico para esse ramo produtivo - Notificação Recomendatória, avaliações individuais dos trabalhadores - e outras abordagens visando intervir nos ambientes com exposição ao benzeno. Resultados: foram observadas alterações em biomarcadores de efeito e de exposição, além de inadequações dos PRC no monitoramento clínico e laboratorial dos trabalhadores. Nos ambientes e processos de trabalho ficaram evidentes situações que ampliam a exposição: inadequações nas pistas de abastecimento, uso de "flanela", inexistência ou insuficiência de medidas de proteção coletivas, ausência de medição eletrônica dos tanques e de higienização dos uniformes por parte das empresas, dentre outras. Conclusão: as experiências desencadeadas por meio de ações integradas no setor saúde demonstraram a potencialidade benéfica da vigilância da exposição ao benzeno em PRC.

Palavras-chave: benzeno; saúde do trabalhador; postos de combustíveis; vigilância.

\begin{abstract}
Introduction: Benzene is an important source of environmental and occupational contamination and may cause poisoning to gas station workers. Objective: to report the benzene exposure surveillance experience carried out by the Centro de Referência em Saúde do Trabalhador (Occupational Health Reference Center) of Região dos Vales, in Rio Grande do Sul, Brazil. Methods: surveillance activities included regional mapping of gas stations, intrasectoral, intersectoral and inter-institutional actions, workplace inspections, development of a specific interventional instrument for this productive branch, and other approaches aiming to intervene in benzene exposure environments. Results: we observed alterations in biomarkers of effect and of exposure, besides companies inadequacies in clinical and laboratory monitoring of workers. In the work processes and the work environments inspected, situations that enlarged exposure were evident: inadequacies in fuel supply lanes, use of cloth strip for fuel leakage absorbance during tank filling, lack or shortage of collective protection measures, absence of volume electronic measurement of the storage tanks and absence of work cloths washing by the companies, among others. Conclusion: experiences unleashed through integrated actions in the health sector demonstrated the beneficial potentiality of the benzene exposure surveillance in gas stations.
\end{abstract}

Keywords: benzene; occupational health; gas stations; surveillance. 


\section{Introdução}

Os solventes orgânicos são substâncias químicas amplamente utilizadas pela sociedade e estão presentes como matéria-prima e/ou produto final em diversos ramos produtivos, o que expõe grande quantidade de trabalhadores a riscos decorrentes do contato direto e indireto com essas substâncias, o que também representa riscos ao meio ambiente e, consequentemente, à população em geral. Entre os solventes temos o benzeno, substância reconhecida como carcinogênica e hematotóxica, cujos efeitos à saúde por exposição têm sido associados com o desenvolvimento de câncer no sistema hematopoiético ${ }^{1}$.

A exposição ao benzeno é ubíqua, pois este solvente é contaminante universal, afetando continuamente os trabalhadores, o meio ambiente e a população em geral. Por essa exposição ser evitável, e também por caracterizar-se em matéria-prima sem perspectiva de substituição ou banimento, amplia-se a necessidade de controle e intervenção sobre o uso e consumo do benzeno ${ }^{2,3}$. A vigilância da exposição ao benzeno e a mitigação dos riscos relacionados ao seu uso é mundialmente recomendada por organismos internacionais, como a International Agency for Research on Cancer (IARC) ${ }^{4}$, órgão da World Health Organization (WHO) $)^{5}$ e, no Brasil, pelo Ministério da Saúde (MS) ${ }^{6}$ e pelo Ministério do Trabalho (MTb) ${ }^{7}$.

O benzeno, além de amplamente utilizado na indústria, também está presente na composição da gasolina, com valor máximo de concentração de 1\% $(\mathrm{v} / \mathrm{v})^{8}$, o que o caracteriza como uma importante fonte de exposição ocupacional e ambiental. Trabalhadores de Postos de Revenda de Combustíveis (PRC) estão expostos ao benzeno devido à emissão de hidrocarbonetos aromáticos voláteis no processo de trabalho, o que pode ocasionar intoxicação pelas vias cutânea, digestiva e, em especial, respiratória. Costa ${ }^{9}$ evidenciou que trabalhadores, durante o abastecimento de gasolina, estavam expostos a concentrações de benzeno no ar que variavam de 40 a $700 \mathrm{ppb}$, na zona respiratória. Apesar de a concentração de benzeno na gasolina ter sido reduzida ao longo dos anos, o benzeno pode estar associado à genotoxicidade e, portanto, contribuir para o desenvolvimento de carcinogenicidade ${ }^{4}$. Mesmo em exposições inferiores a 1 ppm, seus efeitos tóxicos podem levar à formação de metabólitos que desencadeiam a mielotoxicidade, com alterações nas células sanguíneas e leucemogênese ${ }^{10}$. Diante da gravidade da exposição ao benzeno e dos seus efeitos carcinogênicos, os PRCs têm sido alvos de ações de vigilância à saúde.

No Brasil, a vigilância da exposição ao benzeno em PRC foi uma estratégia nacional adotada pela área técnica de saúde do trabalhador do MS que abrangeu diversos Centros de Referência em Saúde do Trabalhador (Cerest) na constituição de uma ação descentralizada de vigilância por ramo produtivo, em vários municípios do país. Os PRC, por estarem presentes na maioria dos municípios, configuram-se como ambientes de trabalho passíveis de vigilância de exposição ao benzeno por equipes locais de profissionais de saúde e meio ambiente, dentre outros. As ações de Vigilância em Saúde (Visa) nesse setor produtivo visam assegurar condições de segurança e saúde para os trabalhadores, estendendo-se à população que reside e/ou trabalha na área de seu entorno, uma vez que o benzeno e outros solventes presentes nos combustíveis e na gasolina geram vapores podendo conter altas concentrações desses compostos, que compartilham rotas metabólicas e de toxificação similares, potencializando os riscos à saúde ${ }^{11,12}$.

O planejamento da Vigilância em Saúde do Trabalhador (Visat), no âmbito da exposição a solventes em postos de combustíveis, buscou efetivar princípios da Política Nacional de Saúde do Trabalhador e da Trabalhadora, que tem como pressupostos o desenvolvimento de ação intersetorial, interinstitucional e também a integração pela articulação de ações intrasetoriais dos vários componentes da Visa (epidemiologia, sanitária, ambiental e saúde do trabalhador), considerando sua complexidade no processo saúde-doença ${ }^{2,13}$. Essa modalidade de vigilância é entendida como um conjunto de práticas integradas e articuladas de intervenção que visam à melhoria da qualidade de vida no trabalho, com participação dos trabalhadores e suas organizações em todas as etapas da vigilância ${ }^{14}$. No que tange à vigilância da exposição, pressupõem-se a participação e mobilização de técnicos e trabalhadores no sentido de intervirem no processo de trabalho e a atividade determinante da exposição. O modelo de vigilância constituído para a área parte da compreensão de que a situação de risco ou do agravo, ou mesmo da conjunção de ambas as situações, se fortalece quando integrada à noção de território e de ação interinstitucional, envolvendo campos de forças com as diferentes expertises resultantes da participação de diversas instituições e atores da temática da vigilância, cujo foco é o processo de trabalho².

Entretanto, apesar das normas e priorização pela vigilância da exposição ocupacional ao benzeno, este grave problema de saúde pública continua sendo um desafio aos serviços de saúde, devido à complexidade da intervenção e do monitoramento dessa exposição. Além disso, a modalidade de vigilância preconizada exige dos profissionais o planejamento de ações que considerem a integralidade e intersetorialidade para a construção de estratégias de prevenção, visando à redução das fontes de exposição, à proteção à saúde dos trabalhadores e do meio ambiente, e à extensão de tais benefícios à população do entorno. Outro 
problema frequente, elencado por Moura-Correa e Santana ${ }^{15}$, refere-se à análise de dados, prática que requer conhecimento e habilidade no manuseio dos dados para gerarem informações com as quais se definem prioridades, objetos específicos para intervenção nos ambientes de trabalho, visando à prevenção da exposição ao benzeno e dos seus efeitos sobre a saúde dos trabalhadores.

Assim, o objetivo deste texto é relatar as potencialidades da vigilância da exposição ao benzeno e a outros agentes químicos, a partir da experiência do Cerest da região dos Vales, no Rio Grande do Sul, na atuação com os PRC, visando contribuir com a vigilância das exposições químicas no Sistema Único de Saúde (SUS).

\section{Métodos}

Trata-se de um relato de experiência que descreve as fases em que foi desenvolvida a vigilância da exposição ao benzeno e a outros agentes químicos presentes nos ambientes de trabalho dos PRC da região dos Vales, área de abrangência do Cerest com sede no município de Santa Cruz do Sul, do estado do Rio Grande do Sul, no período de 2009 a 2015.

A descrição das quatro fases em que se constituem as experiências foi apresentada em sequência cronológica e sistematizada a partir da prática do serviço: a) Fase 1 (Etapa preliminar) que inclui a capacitação e mapeamento dos riscos; b) Fase 2 (Etapa da $1^{\mathrm{a}}$ experiência) período em que as ações de monitoramento da exposição ao benzeno e avaliação dos expostos são desencadeadas no território, no âmbito do município de Santa Cruz do Sul; c) Fase 3 (Etapa da $2^{\text {a }}$ experiência) ação ampliada pela abordagem em território regional; e d) Fase 4 (Etapa da $3^{\mathrm{a}}$ experiência) é representada pela síntese do conhecimento que é reproduzido em publicações e formação, constituindo-se em estratégia de comunicação de risco.

As fases e os resultados das experiências são evidenciados, a partir destas três categorias que fundamentam a ação de vigilância da exposição ao benzeno em PRC: 1) Assistência e análise dos exames (enquanto potencial para evento sentinela); 2) Vigilância de processo de trabalho e 3) Formação e informação para vigilância.

As fontes de dados provêm de duas bases de dados: a Relação Anual de Informações Sociais (RAIS), do MTb, e o Sistema de Informações em Saúde do Trabalhador (SIST/RS), da Secretaria Estadual da Saúde do Rio Grande do Sul. Além desses dados, também foram utilizadas informações de dados gerenciais dos PRC como Programa de Prevenção de Riscos Ambientais (PPRA), Programa de Controle Médico de Saúde Ocupacional (PCMSO), alvarás e Programa de Prevenção e Proteção contra Incêndio. Para a descrição da população de potenciais exposições ao benzeno em PRC, da região dos Vales, utilizou-se a base de dados da RAIS, do Programa de Disseminação das Estatísticas do Trabalho (PDET), disponível para acesso on-line na página do $\mathrm{MTb}^{16}$. Os dados de morbidade dos trabalhadores foram obtidos, no SIST/RS ${ }^{17}$, utilizando o Sistema de Análise de Negócios (SAN), do estado do Rio Grande do Sul.

Os resultados dos exames das avaliações individuais dos trabalhadores provêm dos prontuários do serviço, enquanto os dados de inspeção da ação de vigilância advêm de instrumentos de trabalho do Cerest/Vales. Os formulários empregados para essa coleta de dados foram, respectivamente, a anamnese ocupacional e o Roteiro de Inspeção Sanitária de Ambientes e Processo de Trabalho em Postos de Revenda de Combustíveis (RIAT-PRC) desenvolvido pelo Centro de Estudos da Saúde do Trabalhador (Cesat) da Bahia ${ }^{18}$.

As variáveis descritoras foram municípios da região de abrangência do Cerest/Vales vistoriados e doenças e acidentes relacionados ao trabalho, para os quais se utilizou de resultados dos diagnósticos clínicos e exames laboratoriais dos trabalhadores. Para a categorização das variáveis empregou-se a apresentação descritiva dos municípios. Para as doenças e os acidentes foi empregada a Classificação Internacional de Doenças (CID-10) ${ }^{19}$, compreendendo os capítulos II - Neoplasias (C00-D48), III - Doenças do sangue e dos órgãos hematopoiéticos e alguns transtornos imunitários (D50-D89) e XIX - Lesões, envenenamento e algumas outras consequências de causas externas (S00-T98). Os exames foram classificados em variáveis dicotômicas: realizou exames (Sim/Não) e exames alterados (Sim/Não). Foram excluídos os trabalhadores que, embora tenham realizado avaliação clínica, não entregaram e/ou não fizeram os exames solicitados. A análise dos dados quantitativos foi apresentada por meio da estatística descritiva, no formato de tabela, com distribuição de frequência absoluta e percentual. Diagramas foram utilizados para descrever as fases das experiências de vigilância.

A etapa de vigilância do Cerest/Vales, vinculada à pesquisa estadual, foi registrada no Sistema Nacional de Ética em Pesquisa (Sisnep) e aprovada pelo Comitê de Ética em Pesquisa da Escola de Saúde Pública do Estado do Rio Grande do Sul (Protocolo n ${ }^{\circ}$ 662/11).

\section{Abordagem da Vigilância da exposição ao benzeno}

Devido às diversas ações planejadas para a vigilância da exposição ao benzeno em PRC, apresentam-se as experiências a partir das fases e conteúdos organizativos. 


\section{Fase 1: etapa preliminar}

O curso de Aperfeiçoamento em Vigilância em Saúde da exposição a solventes em postos de combustíveis - região Sul, promovido pela Escola de Saúde Pública do Rio Grande do Sul em parceria com o Centro de Estudos de Saúde do Trabalhador e Ecologia Humana (Cesteh) da Fiocruz e área técnica do MS, foi parte do projeto que desencadeou a vigilância nos PRC no estado do Rio Grande do Sul, em que o Cerest/Vales integrava o grupo de instituições parceiras e constituía a área de abrangência da intervenção. A modalidade do curso estruturou-se como método articulador da formação em serviço, ao integrar a produção do conhecimento e a intervenção no âmbito da vigilância, a partir de trabalho de campo para identificação de risco e utilização do roteiro de inspeção.

\section{Visita técnica e mapeamento}

$\mathrm{Na}$ fase preliminar, o Cerest/Vales iniciou a aplicação dos conhecimentos do curso mediante uma atividade prática com uma visita técnica a um PRC localizado no município de Santa Cruz do Sul. Aliadas à avaliação in loco, foram desenvolvidas outras abordagens: mapeamento da distribuição dos PRC e trabalhadores na região dos Vales, identificação da representação sindical e análise de notificações de agravos relacionados ao trabalho entre trabalhadores desse ramo produtivo. Concluída essa etapa e com o compartilhamento dos conhecimentos com o conjunto de trabalhadores do Cerest/Vales, o serviço assumiu a temática do "benzeno" como prioritária, incorporando-a em todos os eixos de atuação.

Para o mapeamento das instituições, entidades e órgãos públicos com interface de atuação nesse campo, foram contatados os Sindicatos de Trabalhadores, a Unidade Municipal de Referência em Saúde do Trabalhador de Santa Cruz do Sul, o Departamento de Vigilância e Ações em Saúde de Santa Cruz do Sul e a Secretaria de Meio Ambiente de Santa Cruz do Sul.

\section{Ação de vigilância a partir da visita técnica}

A vigilância da exposição ao benzeno em PRC foi realizada por três técnicos do Cerest/Vales (médica do trabalho, técnico de segurança no trabalho e fisioterapeuta), e consistiu, inicialmente, em estudo do processo de trabalho e dos riscos ocupacionais.

Também contribuiu para a inspeção in loco a experiência dos profissionais em vigilâncias noutros ramos produtivos, o roteiro de inspeção, ferramenta que contempla questões fechadas e abertas (que podiam ser complementadas por meio de observação do processo de trabalho e entrevistas não estruturadas com trabalhadores de diferentes funções), registros fotográficos e verificação de documentos administrativos. O roteiro utilizado permitiu identificar aspectos relacionados às avaliações ambientais e individuais dos trabalhadores, no total de 156 questões que abordavam aspectos sobre o ramo de atividade econômica, a operacionalização do abastecimento, o porte dos estabelecimentos, a classificação de operações, os equipamentos, as áreas de trabalho, a força de trabalho, as medidas de saúde e segurança, as exposições, os acidentes de trabalho e o gerenciamento de riscos. Dos documentos administrativos, foram selecionadas informações sobre a situação da saúde dos trabalhadores, os acidentes, os vazamentos e as medidas preventivas adotadas. No Relatório da Vigilância foram registradas as observações dos profissionais, bem como as medidas de adequações sugeridas para a melhoria das condições de trabalho. Essas ações de vigilância foram embasadas nas recomendações técnicas e prescrições legais do MS, sendo que para a inspeção do ambiente de trabalho utilizou-se da instrução normativa da Visat do SUS ${ }^{7}$. Para avaliação dos trabalhadores, a referência foi o protocolo de procedimentos relativos à vigilância da saúde dos trabalhadores expostos ao benzeno ${ }^{20}$.

\section{Fase 2: Primeira experiência - vigilância da exposi- ção em Santa Cruz do Sul}

A primeira experiência do serviço configurou-se como vigilância da exposição, realização de inspeções em outros PRC e avaliações de trabalhadores pela equipe multidisciplinar do Cerest/Vales. Essa experiência evidenciou problemas comuns entre os PRC e motivou a criação de instrumento de intervenção para o ramo produtivo no município, norteando a melhoria nos ambientes e processos de trabalho, bem como o monitoramento biológico dos trabalhadores expostos.

\section{Vigilância da exposição ao benzeno em PRC}

O planejamento dessa vigilância fez parte de um Projeto Estadual de Vigilância da Exposição ao Benzeno em PRC, organizado pela Divisão de Vigilância em Saúde do Trabalhador (DVST), da Secretaria Estadual da Saúde do Rio Grande do Sul (SES/RS). Nele foram contempladas avaliações clínica e laboratorial da potencial exposição e a vigilância do ambiente e do processo de trabalho. Além disso, integrou o projeto um estudo sobre biomarcadores para detecção de alterações precoces em sistemas hematológicos e imunológicos de trabalhadores em PRC, realizado por meio de parceria com a Universidade Federal do Rio Grande do Sul.

Todas as etapas de avaliações dos trabalhadores e vistorias foram executadas por profissionais do Cerest/Vales. 
Mapeamento dos PRC

A partir da listagem de postos de combustíveis registrados na Secretaria Municipal de Fazenda de Santa Cruz do Sul foram identificados os estabelecimentos com maior arrecadação, assumindo que tal condição significa maior exposição da população e do meio ambiente, devido ao quantitativo de produto armazenado e sua comercialização. Além desse critério, associou-se também a paridade entre PRC com diferentes bandeiras e os sem bandeira, para garantir que não ocorresse "sobrecarga" em determinados portes de estabelecimentos.

\section{Instrumentos adotados}

Quanto à inspeção, adotou-se o Roteiro de Inspeção Sanitária de Ambientes e Processo de Trabalho (RIATPRC), formulado pelo Cesat ${ }^{14}$, porém sem a exigência de fornecimento de cópias de documentos à equipe de vigilância. Em relação ao número de trabalhadores a serem submetidos à avaliação clínico-laboratorial, estipulou-se fixar entre 30 e 50\% do quadro funcional, distribuídos nas diferentes funções (gerente, frentista, lubrificador, caixa, chefe de pista, lavador etc.). A avaliação clínica consistiu em acolhimento, consulta médica e avaliação audiológica. $\mathrm{O}$ agendamento foi realizado pelo Cerest/Vales, com vários horários disponibilizados à gerência dos PRC. Durante o acolhimento, houve a solicitação de concordância do trabalhador em se submeter aos procedimentos, mediante assinatura de Termo de Consentimento Informado Livre e Esclarecido. A partir dessa concordância e do conhecimento da jornada de trabalho dos funcionários, planejou-se a realização de exames, com a orientação de que as coletas de marcadores de exposição fossem realizadas ao final da jornada de trabalho.

$\mathrm{Na}$ avaliação multidisciplinar foram utilizadas fichas padronizadas de acolhimento, avaliação audiológica, avaliação clínica com anamnese clínica e ocupacional, elaborada pela equipe dos Cerest regionais e do Cerest estadual do RS. A avaliação médica compreendeu exame físico dirigido e avaliação laboratorial, conforme história individual e relação com a exposição ocupacional (marcadores de efeito e de exposição a solventes).

\section{Fase 3: Segunda experiência - vigilância da exposi- ção (ação integrada)}

A segunda experiência de vigilância da exposição ao benzeno em postos de combustíveis manteve a inspeção in loco e a avaliação individual de trabalhadores. Esta fase se diferenciou pela estratégia de ação de vigilância integrada na região, cuja retaguarda técnica foi assegurada pelo Cerest/Vales com o protagonismo das equipes de saúde dos municípios. Para sua efetivação foi necessário pactuar com técnicos e gestores, realizar formação descentralizada dos profissionais e articular parcerias interinstitucionais.

\section{Qualificação para vigilância da exposição ao benzeno}

Durante o curso de qualificação em Vigilância aos ambientes e processos do trabalho, promovido em duas edições, pelo Cerest/Vales, para profissionais da Visa dos municípios pertencentes à $16^{\mathrm{a}}$ Coordenadoria Regional de Saúde (16 $\left.{ }^{\mathrm{a}} \mathrm{CRS}\right)$, foi proposta a adoção do modelo de vigilância da exposição ao benzeno em PRC para a realização da atividade prática. Para tanto, foi realizada reunião técnica intrasetorial, contando com a participação dos responsáveis na CRS pela Visa (Sanitária, Epidemiológica, Ambiental e Saúde do Trabalhador), pela Atenção Básica e pelo Laboratório Regional do Estado.

\section{Pactuação entre os gestores para vigilância}

A pactuação com gestores foi precedida de apresentação do projeto de Vigilância da exposição ao benzeno pelo Cerest/Vales, sendo esclarecidos os possíveis impactos à saúde dos trabalhadores de PRC, à população do entorno e ao meio ambiente. Com a participação de vinte convidados, pactuou-se a vigilância em oito municípios, da região dos Vales. O pacto incluiu as responsabilidades das instâncias/instituições envolvidas na vigilância. $\mathrm{O}$ Cerest/Vales assumiu a retaguarda técnica na capacitação da equipe municipal, inspeção conjunta do PRC e avaliação dos exames clínicos e laboratoriais. A Secretaria Estadual de Saúde assumiu a responsabilidade pelo custeio da análise bioquímica de marcadores de exposição, que foram realizados pelo Serviço de Análise Ocupacional do Laboratório Central (SAO-LACEN), e pelo apoio às demais ações, conforme a demanda. As secretarias municipais de saúde assumiram a vigilância pela participação das equipes de Visa e da Atenção Básica, da inclusão dos profissionais da área médica, nas capacitações e inspeções, do custeio de análises bioquímicas (marcadores de efeito) e das avaliações clínicas.

Ações de vigilância na região dos Vales

Foram realizadas ações integradas de vigilância da exposição ao benzeno em 12 estabelecimentos de 8 municípios da região dos Vales. As etapas de atividades com os municípios compreenderam: a) compilação de material técnico-informativo e instrumentos para as avaliações; b) realização de capacitação sobre o tema para profissionais de saúde atuantes na Visa e na Atenção Básica; c) pactuação intersetorial quanto a objetivos, estratégias, atribuições e fluxos das equipes; d) realização de ação de vigilância conjunta entre Cerest e Visa municipal e/ou regional; e) realização de avaliação clínica dos trabalhadores dos PRC inspecionados; f) realização de exames laboratoriais: 
minimamente, os marcadores de exposição disponíveis no SAO-LACEN/RS (ácido hipúrico urinário e ácido metil-hipúrico) e os marcadores de efeito disponíveis na rede municipal (hemograma, contagem de plaquetas, prova de função hepática e renal); g) retorno do Cerest/Vales ao município para entrega do Relatório de Vigilância aos Ambientes e Processos de Trabalho; e h) retaguarda técnica, conforme demanda, às equipes da Atenção Básica quanto à avaliação clínica e laboratorial.

\section{Fase 4: Terceira experiência - formação e comunica- ção de risco}

A terceira experiência representa o resultado das anteriores e acrescenta a participação da universidade nas etapas de formação, planejamento e divulgação, o que amplia a comunicação de risco para docentes, acadêmicos, profissionais de saúde, proprietários de postos de combustíveis, dentre outros.

\section{Ampliação das modalidades de capacitação}

Em 2013, com objetivo de qualificar a ação de vigilância da exposição ao benzeno, foi realizado o curso de Atualização em Toxicologia Ocupacional. Nesse curso participaram 18 profissionais de saúde. Em 2014, ocorreu o I Simpósio de Saúde do Trabalhador do Vale do Taquari: Itinerários do Usuário da Atenção Básica, promovido pela Unidade Integrada Vale do Taquari de Ensino Superior (Univates) e pela Secretaria Municipal de Saúde de Lajeado, realizado em junho de 2014.

Na sequência, realizaram-se capacitações para públicos diferenciados (agentes comunitários de saúde, técnicos de enfermagem e demais profissionais da rede de cuidado do Vale do Taquari), incluindo cursos sobre os efeitos da exposição ao benzeno, sobre itinerário da população exposta aos solventes e seus agravos e para qualidade das notificações, nos sistemas de informação em saúde.

As reuniões técnicas entre o Cerest/Vales, a Univates e o setor de Vigilância em Saúde da SMS/ Lajeado precederam a realização do Simpósio - evento que teve o objetivo de efetivar a comunicação de risco com a comunidade científica e a população da região.

\section{Resultados}

O Cerest/Vales, com sede em Santa Cruz do Sul, Rio Grande do Sul, é um serviço do Sistema Único de Saúde (SUS) que integra a Rede Nacional de Atenção Integral à Saúde do Trabalhador (Renast) ${ }^{21}$ do Ministério da Saúde. Esse serviço abrange 68 municípios da região dos vales dos rios Jacuí, Pardo e Taquari, pertencentes à $1^{\mathrm{a}}, 2^{\mathrm{a}}, 6^{\mathrm{a}}, 8^{\mathrm{a}}, 13^{\mathrm{a}}$ e $16^{\mathrm{a}}$
Coordenadorias Regionais de Saúde (CRS) do Rio Grande do $\mathrm{Sul}^{22}$. Em funcionamento desde maio de 2004, o Cerest/Vales conta com equipe multidisciplinar e desenvolve ações nos campos da educação/formação em saúde do trabalhador, vigilância em saúde do trabalhador (epidemiológica e aos ambientes e processos de trabalho), assistência e reabilitação aos trabalhadores portadores de agravos relacionados ao trabalho e de controle social. As ações desenvolvidas pelo Cerest/Vales são exercidas em caráter interinstitucional e intersetorial, visando à integralidade da atenção e ao cumprimento das portarias da Renast ${ }^{21}$.

Na região de abrangência do Cerest/Vales, conforme dados da RAIS, havia 297 estabelecimentos registrados como Comércio varejista de combustíveis para veículos automotores, totalizando 2.458 trabalhadores ou vínculos ativos, em 2013. Trabalhadores de PRC de quatro municípios representam $50 \%$ do total existente na região. O maior número de PRC $(\mathrm{n}=42 ; 14,1 \%)$ e de trabalhadores $(\mathrm{n}=528 ; 21,5 \%)$ da região dos Vales está concentrado em Santa Cruz do Sul, seguido de Lajeado, que possuíam $8,4 \%$ do total de PRC $(n=25)$ e $13,4 \%$ de trabalhadores $(n=329)$. Enquanto em 14 municípios (Cerro Branco, Colinas, Forquetinha, Ibarama, Itapuca, Lagoa Bonita do Sul, Poço das Antas, Pouso Novo, Putinga, Relvado, São Valentim do Sul, Travesseiro, Vale Verde e Vespasiano Corrêa) da região dos Vales havia apenas 1 PRC.

Em relação a acidentes e doenças do trabalho em trabalhadores de PRC, verificou-se na base de dados do SIST/RS o total de 13 registros, entre os quais 12 acidentes, em sua maioria relativos a traumatismos de membro inferior e superior, e 1 caso de anemia aplástica, até agosto de 2015 (Tabela 1).

A experiência da vigilância da exposição ao benzeno, sistematizada na Figura 1, explicita os constituintes dessa ação integrada de vigilância, e a Figura 2 demonstra, a partir da linha do tempo, as etapas e o componente que estruturam o desenvolvimento dessa vigilância, no período de 2009 a 2015. Essas ações foram realizadas pela equipe de vigilância do Cerest/Vales e pelas equipes dos municípios da sua área de abrangência, efetivando a inspeção em 22 PRC e avaliações em 102 trabalhadores.

Essa experiência de vigilância efetivou o total de 1.516 exames (hemograma, plaquetas, provas de função hepática e renal, ácido trans, trans-mucônico, ácido hipúrico e ácido metil-hipúrico) de 102 trabalhadores, pertencentes a nove municípios da região dos Vales, entre 2012 e 2013. Em 2012, período da primeira experiência de vigilância, foram avaliados 50 trabalhadores de PRC de Santa Cruz do Sul. Destas avaliações, verificou-se que pelo menos um dos resultados dos exames dos trabalhadores estava "alterado", quando comparado aos valores 
de referência. Em 2013, etapa da segunda fase, os dados referem-se aos municípios da região dos Vales, período em que se observou que o exame dos trabalhadores de PRC com maior número de alterações foi o ácido hipúrico ( $(n=3)$; enquanto em Santa Cruz do Sul o exame de hemograma obteve maior número $(\mathrm{n}=8)$ de resultados alterados.

Em relação às inspeções realizadas em cinco PRC, no município de Santa Cruz do Sul evidenciaram problemas comuns nos estabelecimentos vigilados, cabendo destacar: 1) não reconhecimento do benzeno como substância cancerígena nas programações de monitoramento da saúde dos trabalhadores; 2) não reconhecimento do risco à saúde auditiva pela exposição a hidrocarbonetos; 3) não adoção de medidas de controle coletivo dos riscos; 4) não informação aos trabalhadores dos riscos à saúde pela exposição aos solventes, especialmente ao benzeno presente nos combustíveis; 5) não realização de exames periódicos que incluíssem marcadores de exposição e de efeito referentes aos riscos presentes nesses locais de trabalho; e 6) dificuldade em coibir comportamentos de risco adotados pelos consumidores na área física dos PRC, como: utilização do telefone celular, consumo de cigarros e bebidas alcoólicas e permanência próxima à área da pista de abastecimento.
No tocante à vigilância realizada em 17 PRC, nos municípios da região dos Vales $(n=9)$, destaca-se: 1) dois não possuíam "bandeira"; 2) apenas um não comercializava álcool combustível; apenas um comercializava gás natural veicular (GNV); 3) dez postos com a pista de abastecimento inadequada; 4) três haviam terceirizado a atividade de lubrificação e quatro a de lavagem; 5) seis não possuíam bomba automática; 6) apenas em três dos postos, a medição dos tanques era realizada eletronicamente; 7) seis armazenavam amostra de combustíveis; 8) somente um estabelecimento dispunha de Ficha de Informações de Segurança de Produtos Químicos (FISPQ) referentes aos produtos utilizados; 9) oito postos forneciam Equipamentos de Proteção Individual (EPI) adequados aos trabalhadores; 10) em todos os estabelecimentos a higienização dos uniformes era realizada pelos próprios trabalhadores; 11) em todos os estabelecimentos a "flanela" era utilizada regularmente pelos frentistas; 12) quatro estabelecimentos não dispunham de PPRA e de PCMSO no local. No que se refere ao monitoramento biológico, somente quatro estabelecimentos realizavam exames periódicos que incluíam avaliação laboratorial. Destes, dois realizavam hemograma, contagem de plaquetas, ácido hipúrico e ácido metil-hipúrico na urina; um realizava hemograma e contagem de plaquetas e um apenas a dosagem de ácido trans, trans-mucônico.

Tabela 1 Distribuição de agravos relacionados ao trabalho no comércio a varejo de combustíveis, conforme município da empresa e tipo de agravo. Região dos Vales*, agosto de 2015

\begin{tabular}{|c|c|c|c|c|c|}
\hline \multirow{2}{*}{ Município } & \multicolumn{2}{|r|}{$D R T^{\text {***** }}$} & \multicolumn{2}{|r|}{ 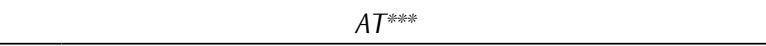 } & \multirow{2}{*}{ Total } \\
\hline & $N$ & CID-10-descritivo & $N$ & CID-10-descritivo & \\
\hline \multirow{2}{*}{ Caçapava do Sul } & - & - & 1 & $\begin{array}{l}\text { S39.1 - Outros traumatismos e os não especificados do } \\
\text { abdome, do dorso e da pelve }\end{array}$ & 2 \\
\hline & - & - & 1 & $\begin{array}{l}\text { S79.1 - Outros traumatismos e os não especificados do } \\
\text { quadril e da coxa }\end{array}$ & \\
\hline Cachoeira do Sul & - & - & 1 & S61.1 - Ferimentos no punho e na mão & 1 \\
\hline Pantano Grande & - & - & 1 & T14.1 - Traumatismo de região não especificada da corpo & 1 \\
\hline Santa Cruz do Sul & 1 & $\begin{array}{c}\text { D61.2 - Anemia aplástica devido } \\
\text { a agentes externos }\end{array}$ & - & - & 1 \\
\hline Doutor Ricardo & - & - & 1 & S90.1 - Traumatismo superficial do tornozelo e do pé & 1 \\
\hline \multirow{3}{*}{ Estrela } & - & - & 1 & S01.1 - Ferimento na cabeça & 3 \\
\hline & - & - & 1 & S60.1 - Traumatismo superficial do punho e da mão & \\
\hline & - & - & 1 & T07.1 - Traumatismos múltiplos não especificados & \\
\hline \multirow{3}{*}{ Lajeado } & - & - & 1 & S01.1 - Ferimento na cabeça & 3 \\
\hline & - & - & 1 & S90.1 - Traumatismo superficial do tornozelo e do pé & \\
\hline & - & - & 1 & $\begin{array}{l}\text { T30.1 - Queimadura e corrosão, parte não especificada } \\
\text { do corpo }\end{array}$ & \\
\hline Teutônia & - & - & 1 & T55.1 - Efeito tóxico de sabões e detergentes & 1 \\
\hline Total & 1 & & 12 & & 13 \\
\hline
\end{tabular}

Fonte: Sistema de Informação em Saúde do Trabalhador - SIST/RS (2015)

*Região dos Vales compreende 68 municípios que compõem a área de abrangência do Cerest/Vales, situada em Santa Cruz do Sul/RS

***: DRT: Doença relacionada ao trabalho

橉AT: Acidente de trabalho 


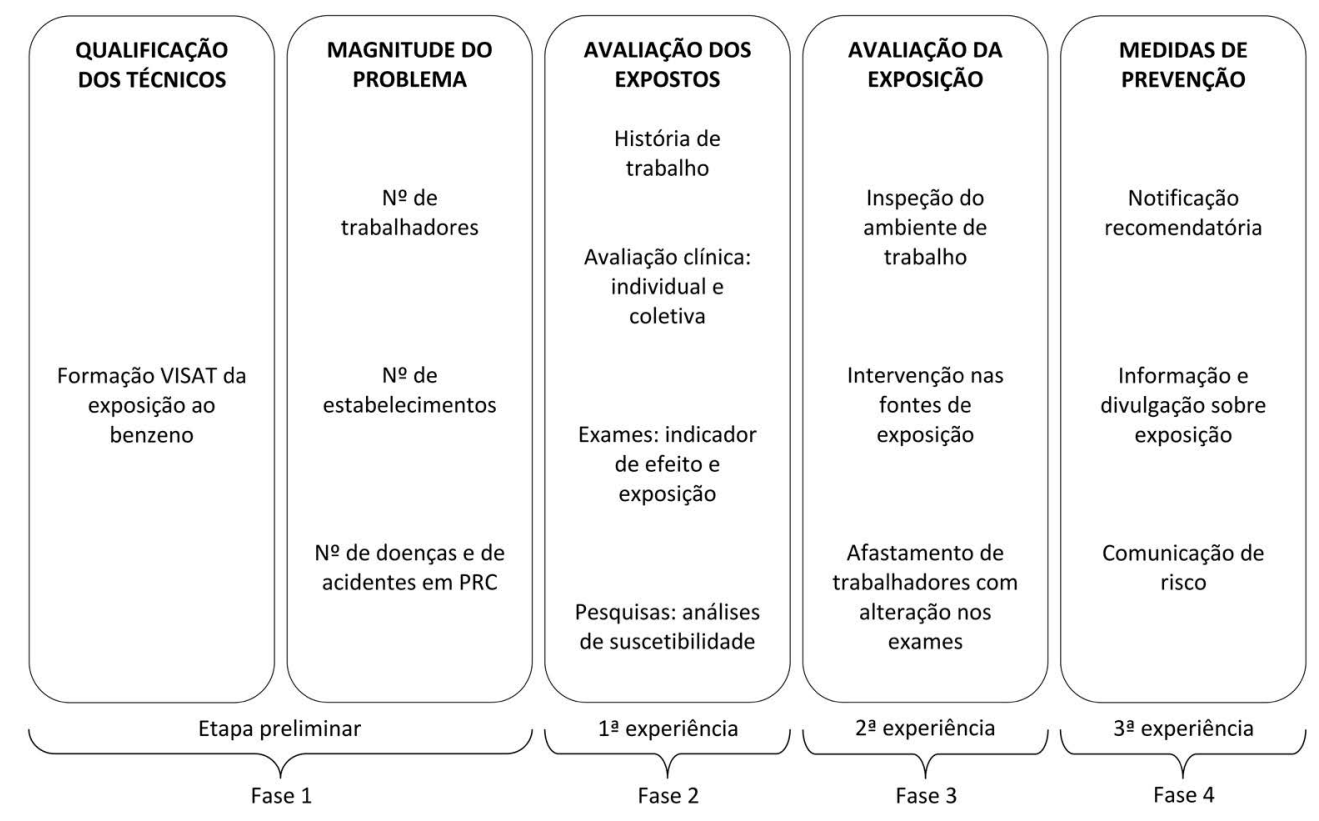

Figura 1 Componentes da ação integrada de vigilância da exposição ao benzeno em Postos de Revenda de Combustíveis (PCR)

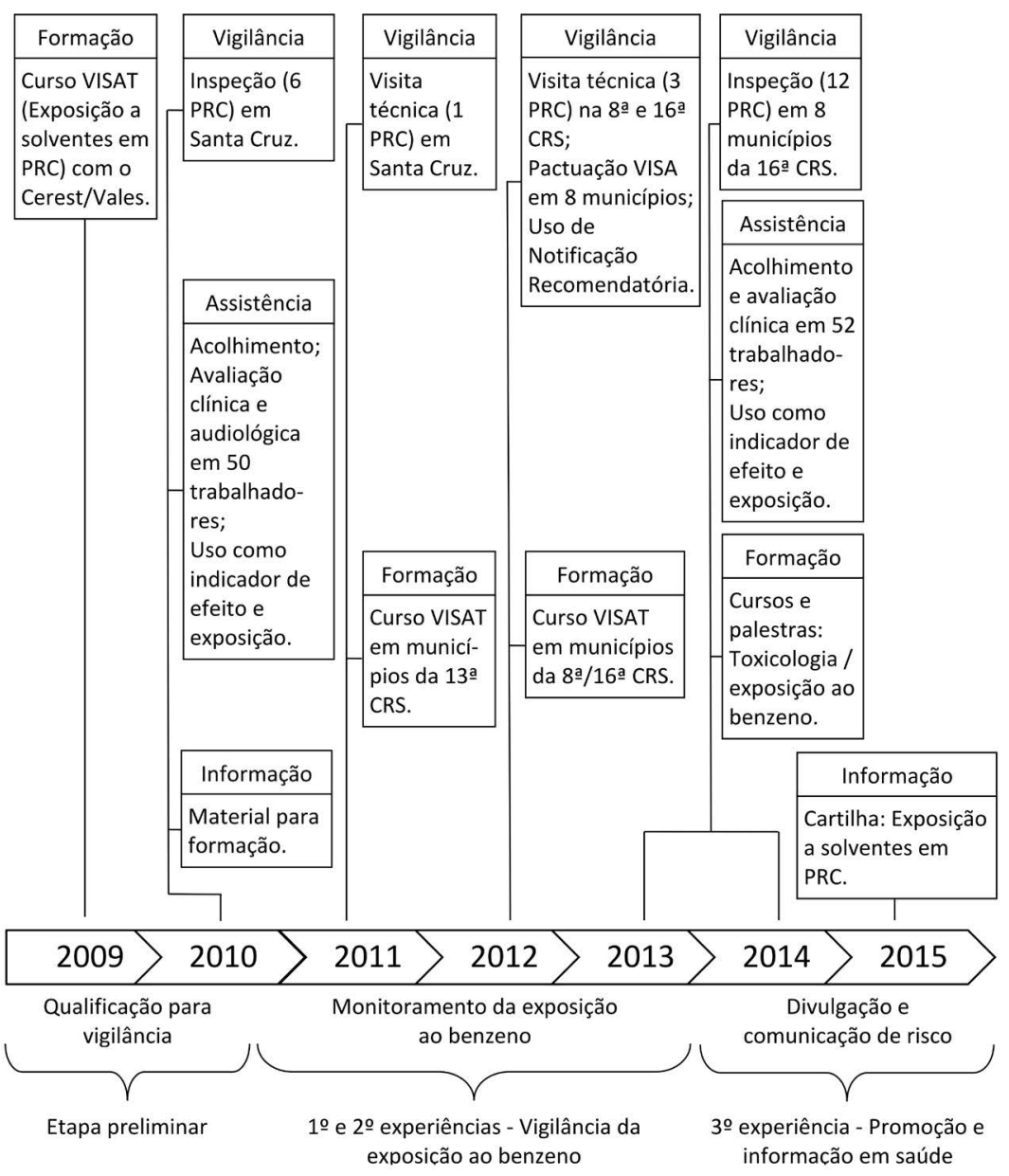

Figura 2 Etapas e componentes da ação integrada de vigilância da exposição ao benzeno em Postos de Revenda de Combustíveis (PCR), na linha do tempo 
O instrumento de Notificação Recomendatória, assinado entre o Cerest/Vales e o Ministério Público do Trabalho, teve como critérios balizadores o evento sentinela e a vigilância por ramo produtivo. Com o entendimento de que é possível potencializar a proteção dos trabalhadores expostos aos solventes em PRC, estabeleceu-se projeção das medidas de correção de risco na fonte e ações preventivas para todos os demais PRC, a partir dos achados nos estudos e ação. Em 2012, a Notificação Recomendatória, direcionada ao PRC, foi detalhada em 24 recomendações, responsabilização de todos empregadores desse ramo produtivo com o controle da exposição e seu monitoramento, no município de Santa Cruz do Sul. Essa notificação envolveu desde a obrigatoriedade ao cumprimento de normas e dos PCMSO e PPRA; o monitoramento biológico dos expostos; as medidas de prevenção de acidentes; as condições seguras e de saúde no ambiente de trabalho, a adoção de equipamentos de proteção individual e coletiva e treinamento dos trabalhadores, procedimentos em situações de acidente, doenças e de emergência, dentre outros.

Em relação à prevenção, percebeu-se a necessidade de esclarecer a trabalhadores, consumidores e profissionais de saúde sobre riscos e formas de prevenção. Para isso, foi elaborado material informativo (folder) com informações sobre solventes; combustíveis; riscos à saúde dos trabalhadores, consumidores e ao meio ambiente; medidas para reduzir riscos aos trabalhadores; e recomendações ao consumidor, incluindo o abastecimento somente até o automático.

Outro desdobramento dessa ação foi a inclusão do tema Vigilância da exposição a solventes/benzeno em PRC nas edições do curso de Qualificação em Vigilância aos Ambientes e Processos do Trabalho, que capacitou 102 profissionais, no período entre 2011 e 2012. A capacitação a partir do I Simpósio de Saúde do Trabalhador do Vale do Taquari envolveu o total de 150 pessoas.

Como resultado do projeto de Vigilância da exposição ao benzeno em PRC, da SES/RS, é importante destacar que estudos de Moro et al. ${ }^{12,23}$, cujo objetivo foi complementar as avaliações em trabalhadores participantes do projeto estadual, incluindo os assistidos pelo Cerest/Vales, com análises de suscetibilidade, verificaram um aumento significativo de danos ao DNA, utilizando o teste Cometa, aumento da frequência de micronúcleos e de alguns indicadores de estresse oxidativo, quando comparados ao grupo controle. Também encontraram redução da atividade da ALA-D, redução da expressão de CD80 e CD86 em monócitos e aumento nos níveis de IL-8 nos trabalhadores avaliados, além de uma redução significativa nos níveis de hemoglobina, contagem de eritrócitos e neutrófilos.

\section{Discussão}

A ação da vigilância, a partir da integração entre assistência, vigilância de processo de trabalho e capacitação direcionada à temática da exposição ao benzeno em PRC, demonstrou ser uma ação bem-sucedida na medida em que consegue apreender os diversos fatores de riscos à saúde presente nesse ambiente de trabalho, integrando ainda abordagens qualitativa e quantitativa de investigação. Constituindo-se, portanto, em uma importante modalidade de vigilância instituída no SUS que pode ser reproduzida em outras situações de exposições químicas. As ações de vigilância abrangeram especialmente os municípios das regiões com maior número de PRC. Foram inseridas também em outros municípios com menores proporções de PRC, conforme planejamento e organização local.

Essa estratégia de vigilância se fortaleceu pela potencialidade da oferta de formação técnica às equipes de vigilância, com desenvolvimento dividido em fases de acúmulo de conhecimento e orientações práticas direcionadas ao monitoramento de um risco específico, o benzeno. A estrutura implementada consolidou um processo que se retroalimenta pela interação entre a formação e a prática e vice-versa, pela possibilidade de experimentações com base na integração de diversas metodologias, conhecimento disciplinares distintos e complementaridade do saber e ação interinstitucional, que repercutiram inclusive na definição de papéis na própria equipe multidisciplinar.

Embora todos os agravos relacionados ao trabalho sejam de notificação compulsória no Rio Grande do Sul e o sistema tenha captado um caso de anemia aplástica, entre os trabalhadores de PRC, esses registros ainda não refletem a realidade da exposição. Certamente, ainda é pouco conhecida dos profissionais de saúde a relação do adoecimento com a exposição ao benzeno, bem como sua notificação como doença do trabalho. Esses achados são importantes para formação em serviços de saúde, no sentido de sensibilizar os profissionais para o diagnóstico e notificação de tais agravos. A inspeção no processo de trabalho evidenciou principalmente que ainda não é reconhecida a carcinogenicidade do benzeno, tanto pelos empregadores quanto os trabalhadores e tampouco são realizadas, na sua totalidade, avaliações e exames de rotina que considerem essa exposição e seus efeitos à saúde.

A experiência de vigilância, em suas fases e ações integradas, se constituiu em fundamental ação para identificação de alterações de biomarcadores e de inadequações nos ambientes de trabalho que reforçam a importância da vigilância da exposição ao benzeno e a outros agentes químicos presentes nos PRC, uma vez que permitem intervir no processo de 
trabalho potencialmente adoecedor antes da ocorrência do agravo. O monitoramento desses trabalhadores pelo SUS e também pelos programas de saúde ocupacional de responsabilidade dos empregadores, recomendado pela equipe durante a ação de vigilância, é uma proposta viável e prevista em normas do Ministério da Saúde. Igualmente importante é a inovação da utilização da Notificação Recomendatória, que utiliza os achados e as recomendações dos ambientes de trabalho dos grupos investigados nos demais estabelecimentos do ramo de atividade econômica, o que operacionaliza a perspectiva de agir a partir de evento sentinela da vigilância em saúde.

Por outro lado, ainda persistem algumas dificuldades, por exemplo, a inexperiência da equipe para intervenções calcadas em exposição complexa, como a do benzeno; a pouca adesão dos trabalhadores expostos à avaliação clínico-laboratorial, possivelmente decorrente da ausência de sintomas e da falta de incentivo para tal por parte dos empregadores. Além disso, a completude de avaliações clínicas dos trabalhadores foi dificultada pela falta de disponibilidade dos biomarcadores de exposição na rede SUS, pela morosidade no recebimento dos resultados de exames laboratoriais e pela alta rotatividade desses trabalhadores, o que consequentemente leva à descontinuidade do monitoramento individual. Essa condição se verificou pelo Indicador Biológico de Exposição para o benzeno, o ácido trans, trans-mucônico - um exame de comprovada sensibilidade e baixa especificidade, em especial para concentrações de benzeno ambiental abaixo de $1 \mathrm{ppm}^{24}$, que, pela alta sensibilidade analítica de sua determinação urinária, apresenta boa correlação com os níveis de benzeno no ar, permitindo diferenciar os expostos e não expostos, caracterização importante para ações de vigilância à saúde. Outra importante limitação foi a ausência da participação da entidade sindical da região nas diversas etapas da vigilância e na elaboração da Notificação Recomendatória.

Ainda assim, essas lacunas não representaram impedimento à efetividade das experiências de Vigilância da exposição ao benzeno, que foi permeada por processo contínuo de apropriação e reprodução do conhecimento teórico e prático sobre a exposição ao benzeno em PRC. Notadamente, a segunda experiência multiplica o conhecimento apreendido com a sensibilização das equipes multiprofissionais na Atenção Básica e na vigilância em saúde dos municípios para a avaliação do impacto à saúde dos trabalhadores nessa atividade econômica, bem como da população do entorno e do meio ambiente.

\section{Conclusão}

Acredita-se que a vigilância integrada e as articulações intra e intersetoriais e interinstitucionais potencializam as ações desenvolvidas pelo Cerest/ Vales, pela possibilidade de ampliar e fortalecer as ações de prevenção à saúde de toda população exposta. Por fim, destaca-se a importância da inclusão desse ramo produtivo no planejamento de ações programáticas do Cerest, do SUS, como indicativo do compromisso institucional assumido em relação à proteção à saúde dos trabalhadores e ao controle do potencial da exposição a carcinógenos em ambientes de trabalho. Pretende-se, a partir dessa experiência da vigilância em PRC, contribuir com um modelo de implantação de monitoramento e avaliação dos trabalhadores expostos ao benzeno.

\section{Contribuições de autoria}

Skamvetsakis A, Santi R e Rocha LHP contribuíram com o relato da primeira experiência (trabalho de conclusão do curso); participaram no mapeamento do ramo produtivo e na articulação com sindicatos de trabalhadores, participaram das visitas técnicas e vigilâncias em ambientes de trabalho, colaboraram com as capacitações das equipes dos municípios, com a elaboração da Notificação Recomendatória. Skamvetsakis A e Santi R elaboraram o material informativo. Brettas FZ colaborou com a avaliação audiológica. Fagundes PS participou do levantamento de dados no Caged e Sist. Skamvetsakis A elaborou as planilhas de dados. Santi R e Brettas FZ contribuíram com a elaboração das planilhas de dados. Skamvetsakis A, Santi R, Brettas FZ e Fagundes PS acolheram e acompanharam os trabalhadores no Cerest/Vales. Skamvetsakis A redigiu o texto, que foi revisado por Brettas FZ, Fagundes PS e Corrêa MJM.

\section{Referências}

1. United States. Department of Health And Human Services. Public Health Service. Agency for Toxic Substances and Disease Registry. Toxicological profile for benzene [Internet]. Atlanta. 2007. [cited
2015 Out 29]. Available from: http://www.atsdr. cdc.gov/toxprofiles/tp3.pdf

2. Machado, JMH, Costa DF, Cardoso LM, Arcuri A. Alternativas e processos de vigilância em 
saúde do trabalhador relacionados à exposição ao benzeno no Brasil. Cienc Saude Colet (online). 2003;8(4):913-21.

3. D’Alascio RG, Menegali M, Bornelli AS, Magajewski F. Sintomas relacionados à exposição ocupacional ao benzeno e hábitos ocupacionais em trabalhadores de postos de revenda de combustíveis a varejo na região Sul de Santa Catarina. Rev Bras Med Trab. 2012;12(1):21-9.

4. International Agency for Research on Cancer (IARC). Monographs on the evaluation of carcinogenic risks to humans. Chemical agents and related occupations [Internet]. Lyon: IARC; 2012 [cited 2015 set 20]. Available from: http:// monographs.iarc.fr/ENG/Monographs/vol100F/

5. World Health Organization (WHO). Preventing disease through healthy environments. Exposure to benzene: a major public health concern [Internet]. Genova: WHO; 2010 [cited 2015 out 29]. Available from: http://www.who.int/ipcs/features/benzene.pdf

6. Brasil. Ministério da Saúde. Secretaria de Atenção à Saúde. Departamento de Ações Programáticas Estratégicas. Risco químico: atenção à saúde dos trabalhadores expostos ao benzeno. Brasília, DF: Editora do Ministério da Saúde; 2006.

7. Brasil. Ministério do Trabalho e Previdência Social. Instrução Normativa 1, 20 de dezembro de 1995. Aprova a vigilância da exposição ocupacional ao benzeno. Diário Oficial [da República Federativa do Brasil]. 4 ago 2011; Seção 1.

8. Brasil. Agência Nacional do Petróleo. Portaria ANP 40, de 25 de outubro de 2013. Estabelece as especificações para a comercialização de gasolinas automotivas em todo o território nacional e obrigações dos agentes econômicos. Diário Oficial [da República Federativa do Brasil]. 30 out 2013.

9. Costa MFB. Estudo da aplicabilidade do ácido trans, trans-mucônico urinário como indicador biológico de exposição ao benzeno [tese]. Rio de Janeiro: Fiocruz; 2001.

10. Smith MT. Advances in understanding benzene health effects and susceptibility. Annu Rev Public Health. 2010;31:133-48.

11. Keenan JJ, Gaffney SH, Galbraith DA, Beatty P, Paustenbach DJ. Gasoline: a complex chemical mixture, or a dangerous vehicle for benzene exposure? Chem Biol Interact. 2010;184(1-2):293-5.

12. Moro AM, Brucker N, Charão MF, Sauer E, Freitas F, Durgante J, et al. Early hematological and immunological alterations in gasoline station attendants exposed to benzene. Environ Res. 2015;137:349-56.

13. Moura-Correa MJ, Jacobina AJ, Santos SA, Pinheiro RD, Menezes MA, Tavares AM, et al. Exposição ao benzeno em postos de revenda de combustíveis no Brasil: Rede de Vigilância em Saúde do Trabalhador (Visat). Cienc Saude Colet (online). 2014;19(12):4637-48.
14. Brasil. Ministério da Saúde. Portaria 3.120 de $1^{\circ}$ de julho de 1998. Aprova a Instrução Normativa de Vigilância em Saúde do Trabalhador no Sistema Único de Saúde. Diário Oficial [da República Federativa do Brasil]. 2 jul 1998; Seção 1:36.

15. Moura-Correa MJ, Santana V S. Matriz de exposição ocupacional potencial: usos e aplicações para estimativas populacionais de expostos ao benzeno. In: Moura-Correa MJ, Pinheiro TMM, Merlo ARC. (organizadores). Vigilância em saúde do trabalhador no Sistema Único de Saúde: teorias e práticas. Belo Horizonte: Coopmed; 2013, p. 125-44.

16. Brasil. Ministério do Trabalho e Previdência Social (MTPS). Relação anual de informações sociais [Internet]. Brasília [acesso 2015 jul 16]. Disponível em: http://acesso.mte.gov.br/portal-pdet/

17. Rio Grande do Sul. Secretaria Estadual de Saúde. Sistema de Informações em Saúde do Trabalhador [Internet]. Rio Grande do Sul [acesso 2015 jul 16]. Disponível em: http://www.saude.rs.gov.br/ lista/324/Vigilância_em_Saúde_do_Trabalhador_ Notificações

18. Bahia. Secretaria da Saúde do Estado da Bahia. Superintendência de Vigilância e Proteção da Saúde. Diretoria de Vigilância e Atenção à Saúde do Trabalhador. Orientações técnicas para ações de vigilância de ambientes e processos de trabalho. Sesab/Suvisa/Divast. Salvador: Divast; 2012.

19. Organização Mundial de Saúde. CID-10: classificação estatística internacional de doenças e problemas relacionados à saúde. 10. rev. São Paulo: Edusp; 1993.

20. Brasil. Ministério da Saúde. Portaria GM/MS 776, de 28 de abril de 2004. Dispõe sobre a regulamentação dos procedimentos relativos à vigilância da saúde dos trabalhadores expostos ao benzeno, e dá outras providências. Diário Oficial [da República Federativa do Brasil]. 29 abr 2004; Seção 1:33.

21. Brasil. Ministério da Saúde. Portaria GM/MS 2.728, de 11 de novembro de 2009. Dispõe sobre a Rede Nacional de Atenção Integral à Saúde do Trabalhador (RENAST) e dá outras providências. Diário Oficial [da República Federativa do Brasil]. 12 nov 2009; Seção 1:76

22. Skamvetsakis A. Gestão compartilhada e humanização em saúde do trabalhador. Bol Saude. 2006;20(2):157-65.

23. Moro AM, Charão MF, Brucker N, Durgante J, Baierle M, Bubols G, et al. Genotoxicity and oxidative stress in gasoline station attendants. Mutat Res. 2013;754(1-2):63-70.

24. Coutrim MX, Carvalho, LRF, Arcuri, ASA. Avaliação dos métodos analíticos para a determinação de metabólitos do benzeno como potenciais biomarcadores de exposição humana ao benzeno no ar. Quím Nova. 2000;23(5):653-63. 